\title{
Symmetry of Magnetically Ordered Quasicrystals
}

\author{
Ron Lifshitz \\ Condensed Matter Physics 114-36, California Institute of Technology, Pasadena, California 91125
}

(Received 1 December 1997)

\begin{abstract}
The notion of magnetic symmetry is reexamined in light of the recent observation of long-range magnetic order in icosahedral quasicrystals [Charrier et al., Phys. Rev. Lett. 78, 4637 (1997)]. The relation between the symmetry of a magnetically ordered (periodic or quasiperiodic) crystal, given in terms of a "spin space group," and its neutron diffraction diagram is established. In doing so, an outline of a symmetry classification scheme for magnetically ordered quasiperiodic crystals, is provided. Predictions are given for the expected diffraction patterns of magnetically ordered icosahedral crystals, provided their symmetry is well described by icosahedral spin space groups. [S0031-9007(98)05584-7]
\end{abstract}

PACS numbers: 75.25.+z, 61.12.Bt, 61.44.Br, 75.50.Kj

Quasicrystals, which today are known to exist in perfectly ordered thermodynamically stable phases, have inspired a reexamination of the basic notions of crystallinity, long-range order, and symmetry [1]. The recent observation by Charrier, Ouladdiaf, and Schmitt [2] of long-range magnetic order in icosahedral quasicrystals of composition $R_{8} \mathrm{Mg}_{42} \mathrm{Zn}_{50}(R=\mathrm{Tb}, \mathrm{Dy}, \mathrm{Ho}, \mathrm{Er})$ is inspiring a similar examination of the nature of magnetic order which, in quasicrystals, had existed previously only as a theoretical construction [3]. The purpose of this Letter is to provide the first steps in this direction by explaining the notion of magnetic symmetry in a manner which applies to both periodic and quasiperiodic crystals, thereby establishing a tool for future analysis of neutron diffraction data and providing a tentative interpretation for the findings of Charrier et al. [2].

We choose to describe a magnetic material by its spindensity field $\mathbf{S}(\mathbf{r})$. This field is a 3-component real-valued function, transforming like an axial vector under $\mathrm{O}(3)$ and changing sign under time inversion. One may think of this function as defining a set of classical magnetic moments, or spins, on the atomic sites of the material. The standard expression (as shown, for example, by Izyumov et al. [4]) for the magnetic contribution to the intensity of elastic scattering of unpolarized neutrons is then given, in terms of the spin-density Fourier coefficients $\mathbf{S}(\mathbf{k})$, as

$$
I(\mathbf{k}) \propto|\mathbf{S}(\mathbf{k})|^{2}-|\hat{\mathbf{k}} \cdot \mathbf{S}(\mathbf{k})|^{2},
$$

where $\mathbf{k}$ is the scattering wave vector and $\hat{\mathbf{k}}$ is a unit vector in its direction.

In making this choice, we follow Litvin and Opechowski [5] who have developed a theory of "spin space groups" to describe the symmetry of periodic magnetic crystals in terms of their spin-density fields. In their theory, symmetry operations are those leaving the magnetic crystal invariant. These are the usual 3-dimensional space-group operations - translations and proper or improper rotations-combined with rotations in "spin space" and possibly also time inversion. We extend their theory here to deal with quasiperiodic crystals which possess neither translational symmetry nor, in general, any rotations that leave them invariant. We choose to extend the theory of spin space groups rather than two other commonly used theories for describing magnetic symmetry-those of color symmetry [6] and the theory of representations of ordinary space groups [7] - because of its direct predictions regarding the outcome of neutron scattering experiments.

What should we expect to see in a neutron diffraction diagram of a single magnetically ordered crystal? To answer this question, we must first clarify what we mean by "crystal." The International Union of Crystallography [8] defines a crystal to be "... any solid with an essentially discrete diffraction diagram." To be more concrete, we consider spin-density fields with well defined Fourier transforms,

$$
\mathbf{S}(\mathbf{r})=\sum_{\mathbf{k} \in L} \mathbf{S}(\mathbf{k}) e^{i \mathbf{k} \cdot \mathbf{r}}
$$

in which the set $L$ contains, at most, a countable infinity of plane waves. In a real experiment, due to the finite resolution of the apparatus, only a finite number of peaks whose intensities $I(\mathbf{k})$ are above a certain threshold will be observed, resulting in a discrete diffraction diagram.

What more can we say about the set of diffraction peaks beyond their being essentially discrete? We have shown elsewhere [9] that if $\mathbf{S}(\mathbf{r})$ describes a physically stable magnetic crystal, i.e., one which minimizes a suitable Gibbs free energy, then the wave vectors $\mathbf{k}$, at which $\mathbf{S}(\mathbf{k}) \neq 0$, are closed under addition and subtraction, with the only exception of peaks that are required by symmetry to vanish. This implies, in practice, that once enough peaks have been observed additional peaks will appear with increased experimental resolution only at integral linear combinations of peaks that already exist.

This leads us to define the set $L$ in Eq. (2) to be the set of all integral linear combinations of the wave vectors $\mathbf{k}$ determined by the diffraction diagram. We call this set the (reciprocal) magnetic lattice of the crystal. The rank $D$ of $L$ is the smallest number of wave vectors needed to generate it by taking integral linear combinations. As described above, we expect that in most (if not all) experimentally observed magnetic crystals this 
number will be finite. Periodic 3-dimensional crystals have a rank $D=3$; aperiodic 3-dimensional crystals have a rank $D>3$; icosahedral quasicrystals, for example, have a rank 6. The first indication of the symmetry of the magnetic crystal is given by the set of (proper or improper) rotations, which, when applied to the origin of Fourier space, merely permute the wave vectors of the magnetic lattice. This set forms a subgroup of $\mathrm{O}(3)$ called the lattice point group $G_{L}$ (also called the holohedry).

The definition of "lattice" here is the same as in nonmagnetic crystals, expect that the magnetic lattice supports the Fourier transform of a vector function $\mathbf{S}(\mathbf{r})$, whereas the lattice of a nonmagnetic crystal supports the Fourier transform of a scalar function - that of the electronic or nuclear density $\rho(\mathbf{r})$ of the crystal. We can, therefore, adopt the symmetry classification scheme, used in the nonmagnetic case, also for magnetic lattices. This classification arranges lattices that have the same rank and the same lattice point group $G_{L}$ into distinct Bravais classes according to the way in which their vectors transform under $G_{L}$ (for details, see, for example, Dräger and Mermin [10]). Icosahedral lattices, for example, are arranged into three Bravais classes [11]: $P$ lattices (primitive) contain all integral linear combinations of the six vectors

$$
\begin{gathered}
\mathbf{v}^{(1,4)}=( \pm 1, \tau, 0), \quad \mathbf{v}^{(2,5)}=(\tau, 0, \pm 1), \\
\mathbf{v}^{(3,6)}=(0, \pm 1, \tau),
\end{gathered}
$$

where $\tau$ is the golden mean; $F^{*}$ lattices (face centered in Fourier space) contain only those combinations in which the sum of the six integers is even; and $I^{*}$ lattices (body centered in Fourier space) contain only those combinations in which the six integers are either all even or all odd.

To say anything further about the nature of the diffraction peaks, we must examine the symmetry of the spin density itself. We reformulate the theory of spin space groups by following the ideas of Rokhsar, Wright, and Mermin's "Fourier-space approach" to crystallography [12]. At the heart of this approach is a redefinition of the concept of 3-dimensional point-group symmetry which enables one to treat quasicrystals directly in 3-dimensional space [13]. The key to redefining point-group symmetry is the observation that certain rotations (proper or improper), when applied to a quasiperiodic crystal, even though they do not leave the crystal invariant, take it into one that contains the same spatial distributions of bounded structures of arbitrary size. One finds that any bounded region in the unrotated crystal is reproduced some distance away in the rotated crystal, but there is, in general, no single translation that brings the two crystals into perfect coincidence.

This weaker notion of symmetry, termed "indistinguishability," is captured by requiring that any symmetry operation of the magnetic crystal leaves invariant all spatially averaged autocorrelation functions of its spindensity field $\mathbf{S}(\mathbf{r})$ for any order and for any choice of components

$$
\begin{aligned}
C_{\alpha_{1} \ldots \alpha_{n}}^{(n)}\left(\mathbf{r}_{1}, \ldots, \mathbf{r}_{n}\right) & \\
& =\lim _{V \rightarrow \infty} \frac{1}{V} \int_{V} d \mathbf{r} S_{\alpha_{1}}\left(\mathbf{r}_{1}-\mathbf{r}\right) \cdots S_{\alpha_{n}}\left(\mathbf{r}_{n}-\mathbf{r}\right) .
\end{aligned}
$$

We have proven elsewhere (see Appendix of Ref. [14]) that an equivalent statement for the indistinguishability of any two quasiperiodic multicomponent fields, in particular two spin density fields $\mathbf{S}(\mathbf{r})$ and $\mathbf{S}^{\prime}(\mathbf{r})$, is that their Fourier coefficients are related by

$$
\mathbf{S}^{\prime}(\mathbf{k})=e^{2 \pi i \chi(\mathbf{k})} \mathbf{S}(\mathbf{k}),
$$

where $\chi$ is a real-valued linear function (modulo integers) on $L$ called a gauge function. Only in the case of periodic crystals can one replace $2 \pi \chi(\mathbf{k})$ by $\mathbf{k} \cdot \mathbf{d}$, reducing indistinguishability to the requirement that the two crystals differ, at most, by a translation $\mathbf{d}$.

We can now define the point group $G$ of the magnetic crystal to be the set of operations $g$ from $\mathrm{O}(3)$ that leave it indistinguishable to within rotations $\gamma$ in spin space, possibly combined with time inversion. Accordingly, for every pair $(g, \gamma)$, there exists a gauge function $\Phi_{g}^{\gamma}(\mathbf{k})$ called a phase function, which satisfies

$$
\mathbf{S}(g \mathbf{k})=e^{2 \pi i \Phi_{g}^{\gamma}(\mathbf{k})} \gamma \mathbf{S}(\mathbf{k}) .
$$

Since $\mathbf{S}([g h] \mathbf{k})=\mathbf{S}(g[h \mathbf{k}])$, one easily establishes that the transformations $\gamma$ in spin space form a group $\Gamma$ and that the pairs $(g, \gamma)$ satisfying the point-group condition (6) form a subgroup of $G \times \Gamma$ which we call the spin point group $G_{\mathbf{S}}$. The corresponding phase functions, one for each pair in $G_{\mathbf{S}}$, must satisfy the group compatibility condition,

$$
\forall(g, \gamma),(h, \eta) \in G_{\mathbf{S}}: \quad \Phi_{g h}^{\gamma \eta}(\mathbf{k}) \equiv \Phi_{g}^{\gamma}(h \mathbf{k})+\Phi_{h}^{\eta}(\mathbf{k}),
$$

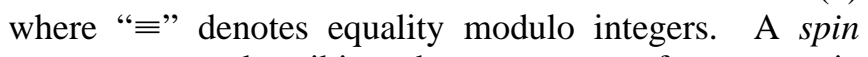
space group, describing the symmetry of a magnetic crystal, whether periodic or aperiodic, is thus given by a magnetic lattice $L$, a spin point group $G_{\mathbf{S}}$, and a set of phase functions $\Phi_{g}^{\gamma}(\mathbf{k})$, satisfying the group compatibility condition (7).

In order to identify further the common symmetry properties of different magnetic structures, whose lattices and spin point groups are equivalent, one classifies their spin space groups into properly chosen equivalence classes called spin space-group types. This is achieved by organizing sets of phase functions satisfying the group compatibility condition (7) into equivalence classes. Two such sets $\Phi$ and $\Phi^{\prime}$ are equivalent if (i) they describe indistinguishable spin-density fields, related as in Eq. (5) by a gauge function $\chi$; or (ii) they correspond to alternative descriptions of the same crystal that differ by their choices of absolute length scales and spatial orientations. In case (i), $\Phi$ and $\Phi^{\prime}$ are related by a gauge transformation:

$$
\forall(g, \gamma) \in G_{\mathbf{S}}: \quad \Phi_{g}^{\prime \gamma}(\mathbf{k}) \equiv \Phi_{g}^{\gamma}(\mathbf{k})+\chi(g \mathbf{k}-\mathbf{k}) .
$$

For a more rigorous definition of these equivalence criteria, see the analogous classification of color space groups (Sec. III of Ref. [14]). 
We said earlier that every wave vector $\mathbf{k}$ in the magnetic lattice $L$ of a magnetic crystal is a candidate for a diffraction peak unless symmetry forbids it. We are now in a position to understand how this happens. Given a wave vector $\mathbf{k} \in L$, we examine all spin point-group operations $(g, \gamma)$ for which $g \mathbf{k}=\mathbf{k}$. These elements form a subgroup of the spin point group which we call the little spin group of $\mathbf{k}, G_{\mathbf{S}}^{\mathbf{k}}$. For elements $(g, \gamma)$ of $G_{\mathbf{S}}^{\mathbf{k}}$, the point-group condition (6) can be rewritten as

$$
\gamma \mathbf{S}(\mathbf{k})=e^{-2 \pi i \Phi_{g}^{\gamma}(\mathbf{k})} \mathbf{S}(\mathbf{k}) .
$$

This implies that the Fourier coefficient $\mathbf{S}(\mathbf{k})$ is required to be a simultaneous eigenvector of all spin transformations $\gamma$ in the little spin group of $\mathbf{k}$, with the eigenvalues given by the corresponding phase functions. If a nontrivial 3-dimensional vector satisfying Eq. (9) does not exist, then $\mathbf{S}(\mathbf{k})$ will necessarily vanish. It should be noted that the phase values in Eq. (9) are independent of the choice of gauge (8) and are, therefore, uniquely determined by the spin space-group type of the crystal.

The process of determining the form of the simultaneous eigenvector $\mathbf{S}(\mathbf{k})$ is greatly simplified if one makes the following observation. Because of the group compatibility condition (7), the set of eigenvalues in Eq. (9) for all of the elements $(g, \gamma) \in G_{\mathbf{S}}^{\mathbf{k}}$ form a 1-dimensional representation of that group. Spin space-group symmetry thus requires the Fourier coefficient $\mathbf{S}(\mathbf{k})$ to transform under a particular 1-dimensional representation of the spin transformations in the little spin group of $\mathbf{k}$. We also independently know that $\mathbf{S}(\mathbf{k})$ transforms under spin rotations as a 3-dimensional axial vector, changing its sign under time inversion. We, therefore, need to check whether the particular 1-dimensional representation, dictated by the spin space group, is contained within the 3-dimensional axialvector representation. If it is not, then $\mathbf{S}(\mathbf{k})$ must vanish; if it is, then $\mathbf{S}(\mathbf{k})$ must lie in the subspace of spin space transforming under this 1-dimensional representation.

Of particular interest are spin transformations $\gamma$ that leave the spin-density field indistinguishable without requiring any rotation in physical space. These transformations are paired in the spin point group with the identity rotation $e$ and form a subgroup of $\Gamma$ called the lattice spin group $\Gamma_{e}$. The lattice spin group plays a key role in determining the outcome of elastic neutron scattering; for if a magnetic crystal has a nontrivial lattice spin group $\Gamma_{e}$, then $\{e\} \times \Gamma_{e} \subseteq G_{\mathbf{S}}^{\mathbf{k}}$ for every $\mathbf{k}$ in the magnetic lattice, restricting the form of the corresponding $\mathbf{S}(\mathbf{k})$. In the special case of periodic crystals, the elements of $\Gamma_{e}$ are spin transformations that when combined with translations leave the magnetic crystal invariant. The phase functions $\Phi_{e}^{\gamma}(\mathbf{k})$, therefore, contain the information which generalizes to the quasiperiodic case the so-called "spin translation groups" of Litvin and Opechowski [5].

What are the possible values of the phase functions $\Phi_{e}^{\gamma}(\mathbf{k})$ ? Consider, for example, a lattice spin group generated by an $n$-fold rotation $\gamma$ about the $z$ axis in spin space, with $n>2$. Repeated applications of the group compatibility condition (7) to $(e, \gamma)^{n}=(e, \epsilon)$, where $\epsilon$ is the identity in spin space, give $0 \equiv \Phi_{e}^{\gamma^{n}}(\mathbf{k}) \equiv n \Phi_{e}^{\gamma}(\mathbf{k})$. Thus, $\Phi_{e}^{\gamma}(\mathbf{k}) \equiv j / n$ for some integer $j$. One can then easily verify through Eq. (9) that

$$
\mathbf{S}(\mathbf{k})= \begin{cases}\left(0,0, S_{z}\right) & \Phi_{e}^{\gamma}(\mathbf{k}) \equiv 0 \\ \left(S_{\perp}, \pm i S_{\perp}, 0\right) & \Phi_{e}^{\gamma}(\mathbf{k}) \equiv \pm \frac{1}{n} \\ (0,0,0) & \text { otherwise }\end{cases}
$$

We have enumerated the distinct lattice spin groups for icosahedral quasicrystals and have found that nontrivial lattice spin groups are possible only with $P$ - or $I^{*}$ magnetic lattices. For each of the lattice spin groups, we have also determined the expected form of $\mathbf{S}(\mathbf{k})$ that is required through (9) by the spin space-group symmetry, for every scattering wave vector $\mathbf{k}$ in the magnetic lattice. These results are summarized in Table I. Further restrictions (not tabulated here) may exist for wave vectors $\mathbf{k}$ lying in the invariant subspaces of nontrivial point-group operations. We emphasize that the diffraction patterns are described here as magnetic lattices with missing points rather than nuclear lattices that are shifted by so-called "magnetic propagation vectors."

Charrier et al. [2] observe magnetic reflections for the $R_{8} \mathrm{Mg}_{42} \mathrm{Zn}_{50}$ quasicrystals at wave vectors of the form $\mathbf{k}=\sum_{i=1}^{6} m_{i} \mathbf{v}^{(i)}+p \mathbf{v}^{(j)}$, where the $\mathbf{v}^{(i)}$ are defined in Eq. (3), the $m_{i}$ are either all even or all odd, $j=1, \ldots, 6$, and $p= \pm \frac{1}{2}$. The nuclear reflections form a body-centered icosahedral (reciprocal) lattice, obtained from the expression above but with $p=0$, corresponding to face-centered ordering in direct space. If the magnetic structure indeed has icosahedral symmetry, and is not merely a collection of magnetic domains in which icosahedral symmetry is broken, then the magnetic lattice, which is formed by taking all integral linear combinations of the observed magnetic reflections, is a primitive icosahedral lattice containing all vectors of the form $\mathbf{k}=\sum_{i=1}^{6} n_{i}\left(\frac{1}{2} \mathbf{v}^{(i)}\right)$ with any integers $n_{i}$. All reflections at wave vectors with $\sum_{i=1}^{6} n_{i}$ even are not observed by Charrier et al. which is consistent with having a lattice spin group $1^{\prime}$, as shown by the first entry for $P$ lattices in Table I. Other peaks that are not observed might just be too weak in the current experiment rather than actually missing due to symmetry requirements. This may suggest that, in direct space, the magnetic structure has an underlying antiferromagnetic body-centered icosahedral ordering (analogous to the chemical ordering in the cubic cesium chloride structure). This may occur, for example, if only a fraction of the rare-earth atoms have their magnetic moments aligned, this fraction arranged in a body-centered icosahedral superstructure made of tiles with twice the edge length. One would need to develop actual models to test such hypotheses.

We have demonstrated that, through its fairly simple selection rules (9), the theory of spin space groups provides a valuable tool for analyzing neutron diffraction 
TABLE I. Icosahedral lattice spin groups and their effect on the outcome of neutron scattering experiments. Nontrivial lattice spin groups are possible only with primitive $(P)$ magnetic (reciprocal) lattices or with body-centered $\left(I^{*}\right)$ magnetic (reciprocal) lattices. The lattice spin groups, which are groups of rotations in spin space, are specified in the leftmost column in terms of their generating rotations, where $2_{i}$ is a twofold rotation about the $i$ axis in spin space $(i=x, y$, or $z), 1^{\prime}$ is the time inversion operation which takes every $\mathbf{S}(\mathbf{k})$ into $-\mathbf{S}(\mathbf{k})$, and $2_{i}^{\prime}$ is the product of the two. The form of $\mathbf{S}(\mathbf{k})$ is given for each scattering wave vector in the magnetic lattice according to its indexing by the six vectors $\mathbf{v}^{(i)}$ defined in Eq. (3). In the case of $I^{*}$ lattices, three different scattering patterns are possible for each lattice spin group depending on the scale chosen for the generating vectors $\mathbf{v}^{(i)}$. Entries in brackets are related to the ones above them through a scaling of the $I^{*}$ lattice by the golden mean. The forms of the $\mathbf{S}(\mathbf{k})$ given in the table may be used in conjunction with expressions, such as Eq. (1), to determine the outcome of neutron scattering experiments.

\begin{tabular}{|c|c|c|c|c|c|c|}
\hline & $P$ lattice & - any integers $n_{i}$ & & $I^{*}$ lattices $-I$ & even or all odd & \\
\hline & $\sum n_{i}=2 n$ & $\sum n_{i}=2 n+1$ & $\begin{array}{l}n_{i} \text { all even } \\
\sum n_{i}=4 n\end{array}$ & $\begin{array}{c}n_{i} \text { all even } \\
\sum n_{i}=4 n+2\end{array}$ & $\begin{array}{c}n_{i} \text { all odd } \\
\sum n_{i}=4 n \\
\end{array}$ & $\begin{array}{c}n_{i} \text { all odd } \\
\sum n_{i}=4 n+2\end{array}$ \\
\hline $1^{\prime}$ & $(0,0,0)$ & $\left(S_{x}, S_{y}, S_{z}\right)$ & {$[(0,0,0)]$} & {$\left[\left(S_{x}, S_{y}, S_{z}\right)\right]$} & {$\left[\left(S_{x}, S_{y}, S_{z}\right)\right]$} & {$[(0,0,0)]$} \\
\hline & & & {$[(0,0,0)]$} & {$\left[\left(S_{x}, S_{y}, S_{z}\right)\right]$} & {$[(0,0,0)]$} & {$\left[\left(S_{x}, S_{y}, S_{z}\right)\right]$} \\
\hline & & & $\left(0,0, S_{z}\right)$ & $\left(0,0, S_{z}\right)$ & $\left(S_{x}, S_{y}, 0\right)$ & $\left(S_{x}, \dot{S}_{y}, 0\right)$ \\
\hline & & & $\left(S_{x}, S_{y}, 0\right)$ & $\left(S_{x}, S_{y}, 0\right)$ & $\left(0,0, S_{z}\right)$ & $\left(0,0, S_{z}\right)$ \\
\hline $2_{z}^{\prime}$ & $\left(S_{x}, S_{y}, 0\right)$ & $\left(0,0, S_{z}\right)$ & {$\left[\left(S_{x}, S_{y}, 0\right)\right]$} & {$\left[\left(0,0, S_{z}\right)\right]$} & {$\left[\left(0,0, S_{z}\right)\right]$} & {$\left[\left(S_{x}, S_{y}, 0\right)\right]$} \\
\hline & & & {$\left[\left(S_{x}, S_{y}, 0\right)\right]$} & {$\left[\left(0,0, S_{z}\right)\right]$} & {$\left[\left(S_{x}, S_{y}, 0\right)\right]$} & {$\left[\left(0,0, S_{z}\right)\right]$} \\
\hline & & & $(0,0,0)$ & $\left(0, S_{y}, 0\right)$ & $\left(S_{x}, 0,0\right)$ & $\left(0,0, S_{z}\right)$ \\
\hline $2_{x} 2_{y} 2_{z}$ & & N.A. & {$[(0,0,0)]$} & {$\left[\left(S_{x}, 0,0\right)\right]$} & {$\left[\left(0,0, S_{z}\right)\right]$} & {$\left[\left(0, S_{y}, 0\right)\right]$} \\
\hline & & & {$[(0,0,0)]$} & {$\left[\left(0,0, S_{z}\right)\right]$} & {$\left[\left(0, S_{y}, 0\right)\right]$} & {$\left[\left(S_{x}, 0,0\right)\right]$} \\
\hline & & & $(0,0,0)$ & $(0,0,0)$ & $\left(0,0, S_{z}\right)$ & $\left(S_{x}, S_{y}, 0\right)$ \\
\hline $2_{z} 1^{\prime}$ & & N.A. & {$[(0,0,0)]$} & {$\left[\left(0,0, S_{z}\right)\right]$} & {$\left[\left(S_{x}, S_{y}, 0\right)\right]$} & {$[(0,0,0)]$} \\
\hline & & & {$[(0,0,0)]$} & {$\left[\left(S_{x}, S_{y}, 0\right)\right]$} & {$[(0,0,0)]$} & {$\left[\left(0,0, S_{z}\right)\right]$} \\
\hline
\end{tabular}

diagrams of either periodic or quasiperiodic magnetically ordered crystals. This is not to say that the use of color symmetry, which has been extended to quasicrystals [14], and the use of representations of ordinary space groups, which have yet to be extended to quasicrystals, will not offer any additional insight.

The author thanks Michael Cross, Veit Elser, David Mermin, Peter Weichman, Benoît Charrier, and Denys Schmitt for valuable discussions, and the latter two, also, for sending their preprints prior to publication. This work was supported by Caltech through a Division Research Fellowship in Theoretical Physics.

[1] For a current textbook on the physics of quasicrystals, see C. Janot, Quasicrystals: A Primer (Clarendon Press, Oxford, 1994), 2nd ed.; for a textbook on the mathematics of quasicrystals, see M. Senechal, Quasicrystals and Geometry (Cambridge University Press, Cambridge, England, 1995).

[2] B. Charrier, B. Ouladdiaf, and D. Schmitt, Phys. Rev. Lett. 78, 4637 (1997).

[3] R. Lifshitz, in Proceedings of the 5th International Conference on Quasicrystals, edited by C. Janot and R. Mosseri (World Scientific, Singapore, 1995), p. 43.

[4] Yu. A. Izyumov, V. N. Naish, and R. P. Ozerov, Neutron Diffraction of Magnetic Materials (Consultants Bureau, New York, 1991), Eq. (17.10).
[5] D. B. Litvin and W. Opechowski, Physica (Amsterdam) 76, 538 (1974); D. B. Litvin, Acta Crystallogr. Sect. A 29, 651 (1973); Acta Crystallogr. Sect. A 33, 279 (1977).

[6] For a review, see, for example, R. L. E. Schwarzenberger, Bull. London Math. Soc. 16, 209 (1984); W. Opechowski, Crystallographic and Metacrystallographic Groups (North-Holland, Amsterdam, 1986).

[7] E. F. Bertaut, Acta Crystallogr. Sect. A 24, 217 (1968).

[8] International Union of Crystallography, Acta Crystallogr. Sect. A 48, 922 (1992).

[9] R. Lifshitz (unpublished). For a sketch of the argument, see Extended Abstracts of the Workshop on Application of Symmetry Analysis to Diffraction Investigation, edited by W. Sikora (University of Mining and Metallurgy, Kraków, Poland, 1996), p. 70.

[10] J. Dräger and N. D. Mermin, Phys. Rev. Lett. 76, 1489 (1996).

[11] D. Rokhsar, N. D. Mermin, and D. Wright, Phys. Rev. B 35, 5487 (1987).

[12] For a review, see N. D. Mermin, Rev. Mod. Phys. 64, 3 (1992); for an elementary introduction, see R. Lifshitz, Physica (Amsterdam) 232A, 633 (1996).

[13] As opposed to the alternative "superspace approach," a review of which is found in T. Janssen, A. Janner, A. Looijenga-Vos, and P.M. de Wolff, in International Tables for Crystallography, edited by A.J.C. Wilson (Kluwer, Dordrecht, 1992), Vol. C, p. 797.

[14] R. Lifshitz, Rev. Mod. Phys. 69, 1181 (1997). 